\title{
SEPARAÇÃO DE PIGMENTOS FOTOSSINTÉTICOS DO FOPLCTON POR MEIO DE CROMATOGRAFIA LIQUIDA DE ALTA EFICIÊNCIA (CLAE)
}

\author{
LUÍS ANTÔNIO PROENÇA* \\ Faculdade de Ciências do Mar - FACIMAR/UNIVALI \\ Rua Uruguai, 458, Itajaí - SC, 88302-202 \\ E-Mail:oceano@melim.com.br
}

\begin{abstract}
RESUMO
Pigmentos de algas são utilizados amplamente em oceanografia sendo que um dos mais importantes índices, o da biomassa do fitoplâncton, baseia-se na determinação da clorofila a (cia a). Diferente de vegetais superiores, uma das características das algas do plancton é a existência de uma grande diversidade de pigmentos, chamados acessórios ou fotossintéticos, que estão distribuídos entre as diferentes classes. Estes pigmentos incluem clorofilas, carotenóides e ficobiliproteínas. O valor destes pigmentos como ferramenta para estudos filogenéticos, taxonômicos, fisiológicos, ecológicos e outros é grande. Embora a determinação de cla a seja relativamente fácil, a determinação de carotenôides é complexa, dado que estes tem seus máximos de absorção próximos, em torno de $450 \mathrm{~nm}$, e por não possuirem fluorescência. Porém, nos últimos anos, com desenvolvimento de novos materiais e métodos, a técnica de cromatografia líquida de alta eficiência (CLAE) tem sido utilizada amplamente para a determinação de pigmentos fotossintéticos em oceanografia. No presente trabalho, são apresentados resultados da separação por CLAE de pigmentos fotossintéticos de 6 representantes de grupos diferentes de algas (dinoflagelados, algas verdes, criptomônadas, primnesiofitas, diatomáceas, e cianof ítas). Os pigmentos foram identificados por meio de seu tempo de retenção e de suas caraterísticas espectrais, em comparação com a composição de pigmentos de algas documentada na literatura. Um total de 19 pigmentos foram identificados. A aplicação da técnica é demonstrada na análise de 3 amostras naturais.
\end{abstract}

Palavras Chave: Pigmentos, Carotenoides, CLAE, Fitoplâncton

\section{DETERMINATION OF ALGAL PHOTOSYNTHETIC PIGMENTS BY HIGH PERFORMANCE LIQUID CHROMATOGRAPHY.}

\begin{abstract}
One of the most important index in oceanography, phytoplankton biomass, is based on determination of chlorophyll a, an algae pigment. Unlike higher plants, algae show in their composition a large number of pigments broadly called accessory or photosynthetic pigments. These pigments include chlorophylls, carotenoids and phycobiliproteins. Measuring the relative or absolute concentration of algae pigments is a powerful tool to study algae phylogeny, taxonomy, physiology, ecology and others. Chlorophyll a concentration is determined in natural samples either by spectroscopy or fluorometry. In the other hand, the determination of other photosynthetic pigments, such as carotenoids, is difficult due to their close absorption characteristic and the absence of fluorescence. In the last few years, with new materials and technologies development, chromatographic methods, specially high performance liquid chromatography (HPLC), have been successfully applied to quantify chlorophylls and carotenoids in natural waters. These new methodologies opened a new perspective on measuring algae pigments, either by improving the determination of chlorophyll a precision or by making possible to measure up to 50 pigments, including some degradation products. In this paper, a procedure to qualitatively calibrate an HPLC system is presented. Nineteen pigments, from six species, representing different algae groups (dinoflagellates, green algae, cryptomonads, prymnesiophytes, diatoms and cyanophytes) were separated and identified by their retention times and spectral characteristics. The
\end{abstract}


method application is exemplified in 3 natural samples.

Key-words: Pigments, Carotenoids, HPLC, Phytoplankton.

* Bolsista CNPq

\section{INTRODUÇÃO}

Pigmentos de algas são utilizados amplamente em oceanografia, sendo que um dos mais importantes índices, o da biomassa do fitoplâncton, baseia-se na determinação da clorofila a (cla a.) A popularidade da cia a como indicador da biomassa do fitoplâncton deriva do fato de esta ser exclusiva de organismos autotróficos fotossintetisantes e ser sua estimativa relativamente fácil em amostras naturais. Desta forma, a cia a faz parte daquelas variáveis básicas empregadas para descrever os ecossistemas aquáticos. Diferente de vegetais superiores, uma das características das algas do plâncton é a existência de uma grande diversidade de pigmentos chamados acessórios ou fotossintéticos, distribuídos entre as diferentes classes. $O$ valor destes pigmentos como critério para estudos filogenéticos e taxonômicos é grande e sua aplicação ocorre desde o começo do estudo da composição de pigmentos por classes ou grupos de algas (Rowan, 1989). Além das clorofilas, que somam aproximadamente 10 tipos conhecidos e das ficobiliproteinas, pigmentos solúveis em água, as algas apresentam um grande número de carotenóides. Os carotenóides são pigmentos lipossolúveis divididos em dois tipos, os carotenos e xantofilas. Os carotenos são poucos em número, enquanto as xantofilas, também hidrocarbonos, porém com ao menos um átomo de oxigênio na molécula, perfazem o maior número dos carotenóides. Duas funções principais têm sido atribuídas aos carotenóides: captação de energia luminosa contida nos diferentes comprimentos de onda da radiação e proteção da cia a ativada contra o processo de foto-oxidação (Rowan,1989).

A determinação de cia a em amostras naturais baseia-se ou em seu máximo de absorção ou em suas propriedades fluorescentes por meio dos métodos espectrofotométrico ou fluorescência, respectivamente (Parsons et al. 1984). A determinação de carotenóides, por sua vez, é mais complexadado que estes não possuem fluorescência e têm seus máximos de absorção próximos, em torno de $450 \mathrm{~nm}$, tornando difícil sua identificação e quantificação por métodos convencionais de espectrofotometria. Desta forma, estudos sobre outros pigmentos fotossintéticos em oceanografia, além da cia a, são mais restritos. Porém, nos últimos anos, técnicas de cromatografia para pig-mentos vegetais têm sido aperfeiçoadas, principalmente a técnica de cromatografia líquida de alta eficiência (CLAE). A otimização da CLAE para pigmentos é recente e possibilitou seu emprego mais generalizado. Análises que antes demoravam horas, hoje podem ser feitas em alguns minutos com bastante precisão. Com o emprego das técnicas modernas de cromatografia, informações pertinentes a distribuição de pigmentos, principalmente clorofilas e carotenóides, tornaram-se mais disponíveis. Estas informações abrem várias perspectivas para os estudos oceanográficos (p. ex. Giekes \& Kraay, 1983; Everitt et. al., 1990; Barlow et.al. 1993; Bianchi et al. 1993). A CLAE, também chamada de alta pressão, como conhecemos hoje, foi desenvolvida no final da década de 60 . A partir da cromatografia de coluna, na qual a fase móvel (solventes) percolava através da fase estacionária (sólida) pela força da gravidade, adaptações foram feitas no sentido de forçar, por meio de bombeamento, a passagem da fase móvel. Com isto, foi possível aumentar a velocidade de fluxo, e consequentemente da cromatografia, e reduzir o tamanho das partículas da fase estacionária, aumentando a eficiência da separação cromatográfica. Como 
resultado, a pressão do sistema aumentou e foram necessários várias adaptações, as quais foram possíveis com o desenvolvimento de novos materiais (Braithwite e Smith, 1985).

Para a determinacão de pigmentos fotossintéticos em amostras naturias é necessária a calibração do sistema cromatográfico. A calibração é feita em dois passos. O primeiro, qualitativo, refere-se a otimização do sistema e obtenção dos tempos de retenção dos pigmentos a serem analisados. $O$ segundo, quantitativo, refere-se a calibração da resposta do detector (absorbância ou fluorescência) em relação a quantidade de pigmento injetado. Os padroes de pigmentos disponíveis no mercado são restritos a alguns pigmentos (ex. cia a, clorofila b e alugns carotenos). Uma alternativa para a calibração qualitativa é a utilização de culturas de algas com a composição de pigmentos conhecida (Mantoura e Llewellyn, 1983; Wright et al. 1991).

No presente relatório, o procedimento de implantação e calibração qualitativa de um sistema de cromatografia líquida de alta eficiência é apresentado. Para tanto, foram separados os pigmentos de algas represantando 6 grupos diferentes: dinoflagelados (Amphidinium carterae), algas verdes (Dunaliella tertiolecta), criptomônadas (Hillea $\mathrm{sp}$ ), primnesiofitas (Pavlova luthen), diatomáceas (Phaeodactilum tricomutum) e cianofitas
(Synechococcus subsalsus). Como exemplo da aplicação da técnica, são apresentados resultados da separção de pigmentos contidos em 3 amostras naturais.

Com esta calibração, será possivel a análise rotineira, no Laboratório de Oceanografia Biológica de pigmentos fotossintéticos em amostras naturais do fitoplâncton e macroalgas.

\section{MATERIAL E MÉTODOS}

\section{Sistema de Cromatografia Líquida de Alta Eficiência (CLAE):}

O conjunto de utilizado consistiu em um sistema de CLAE Shimadzu ${ }^{\circledR}$ LC10 composto de: sistema de degassificação por borbulhamento de hélio, uma bomba de alta pressão LC10-AD, capaz de realizar gradientes quaternários, uma válvula seletora de solventes FLD-10A, um detetor de absorbância de fotodiodo SPDM-10A com 512 elementos (resolução de $1 \mathrm{~nm}$ ), para detecção entre 200 e 600 nm, um detetor de fluorescência RF-551 e uma controladora CBM-10A (figura 1). As amostras, diluídas na fase móvel $A$, foram injetadas por meio de um injetor Rheodine ${ }^{\circledR} 7725$ i equipado com um loop de $50 \mathrm{I}$. Os dados gerados durante a cromatografia foram armazenados e processados em microcomputador, utilizando-se

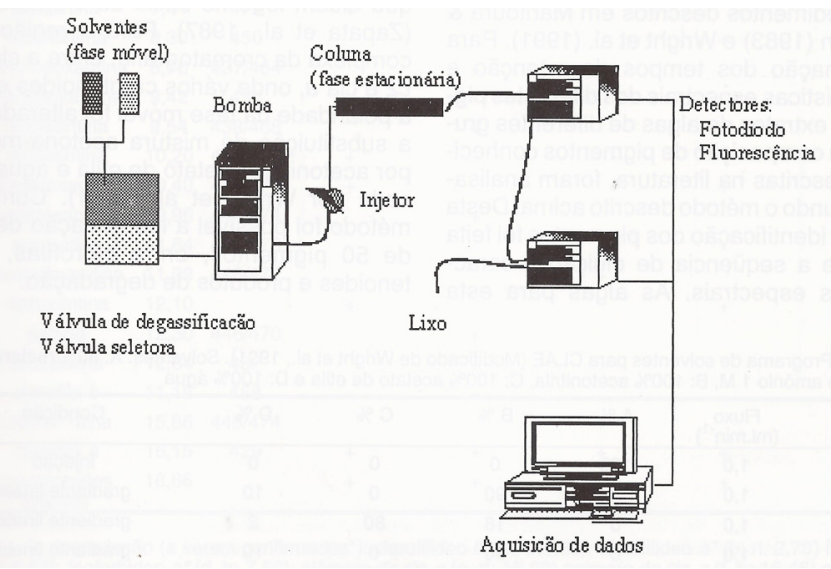

Figura 1 - Esquema do sistema de cromatografia líquida de alta eficiência utilizado 
o programa de aquisição LC10, também da Shimadzu. No presente relatório apresentamos apenas o dados referentes a detecção por absorbância.

A cromatografia ocorreu em uma coluna cromatográfica SHIM-PACK ${ }^{\circledR},(4,6$ $\mathrm{mm}$ de diâmetro interno e $40 \mathrm{~mm}$ de comprimento) empacotada com partículas ODS de $3 \mathrm{~m}$ de diâmetro. $\mathrm{O}$ sistema de gradiente de solventes usado seguiu Wright et al., (1991) (Tabela 1).

Os solventes empregados foram de alta pureza, grau HPLC e a água filtrada em sistema Milli-Q ${ }^{\circledR}$ (Whaters Co.). O acetato de amônia foi de pureza PA.

\section{Extração dos pigmentos:}

A extração dos pigmentos retidos em filtro Whatman GF/F (poro nominal: 0,7 m) foi feita em acetona aquosa $90 \%$ durante 24 horas em frezer. Antes da injeção no cromatógrafo, os extratos foram clarificados por filtração em filtro Whatman GF/F.

\section{Identificação dos Pigmentos:}

A identificação dos pigmentos seguiu os procedimentos descritos em Mantoura \& Llewellyn (1983) e Wright et al. (1991). Para determinação dos tempos de retenção e características espectrais dos diferentes pigmentos, extratos de algas de diferentes grupos com composição de pigmentos conhecidas e descritas na literatura, foram analisados segundo o método descrito acima. Desta forma, a identificação dos pigmentos foi feita em base a seqüencia de eluição e características espectrais. As algas para estaanálise foram obtidas junto ao banco de algas do Instituto Oceanográfico da Universidade de São Paulo. O tempo de retenção da cla a foi obtido por meio da cromatografia de padrão de cia a pura (Sigma Co.). A tabela 2 mostra os diferentes pigmentos encontrados nas diferentes espécies de algas, seus tempos de retenção e os máximos de absorção observados (entre 200 e 600 e diluídos em fase móvel).

\section{RESULTADOS E DISCUSSÃO}

As modificações metodológicas na cromatografia líquida de pigmentos empregadas no começo de década de 80 otimizaram bastante a análise. No trabalho de Mantoura \& Llewellyn (1983), aproximadamente 30 pigmentos puderam ser separados e estimados em uma cromatografia de aproximadamente 20 minutos. Modificações sucessivas foram propostas em base ao método de Mantoura \& Llewellyn, as quais incluíram a substituição do pareador iônico por acetato de amônia para a separação das clorofilas $c$ e clorofilídeos, que eluem logo no início da cromatografia (Zapata et al., 1987). Para a região mais complexa da cromatografia, entre a clorofila c2 e cla a, onde vários carotenoides eluem, a polaridade da fase móvel foi alterada com a substituição da mistura acetona-metanol por acetonitrila-acetato de etila e água, aplicada por Wright et al. (1991). Com este método foi possível a identificação de mais de 50 pigmentos, entre clorofilas, carotenoides e produtos de degradação.

Tabela 1 - Programa de solventes para CLAE (Modificado de Wright et al., 1991). Solventes: A: 80\% metano!, 20\% acetato de amônio $1 \mathrm{M}, \mathrm{B}$ : $100 \%$ acetonitrila, C: $100 \%$ acetato de atila e D: $100 \%$ água

\begin{tabular}{lcccccc}
\hline $\begin{array}{l}\text { Tempo } \\
\text { (minutos) }\end{array}$ & $\begin{array}{c}\text { Fluxo } \\
\left(\mathrm{ml}^{-1} \mathrm{~min}^{-1}\right)\end{array}$ & $\mathrm{A} \%$ & $\mathrm{~B} \%$ & $\mathrm{C} \%$ & $\mathrm{D} \%$ & Condição \\
\hline 0 & 1,0 & 100 & 0 & 0 & 0 & injeção \\
3 & 1,0 & 0 & 90 & 0 & 10 & gradiente linear \\
18 & 1,0 & 0 & 18 & 80 & 2 & gradiente linear \\
20 & 1,0 & 0 & 90 & 0 & 10 & gradiente linear \\
30 & 1,0 & 100 & 0 & 0 & 0 & equilibração da coluna \\
\hline
\end{tabular}


O emprego do método de separação descrito em Wright et al. (1991) possibilitou uma boa separação dos pigmentos das algas analisadas. A figura 2 mostra os cromatogramas de absorbância $(440 \mathrm{~nm})$ das diferentes algas analisadas pelo método. Os picos dos cromatogramas correspondem aos números dos pigmentos listados na tabela 2 . Os espectros de absorção, entre 350 e 600 $\mathrm{nm}$, de alguns dos pigmentos separados são mostrados nas figuras 3 e 4 .

Como mostra a figura 4 , as características espectrais de alguns carotenóides podem ser bastante semelhantes, como ocorre, por exemplo, entre diadinoxantina e luteína. Neste caso, a identificação baseiase no tempos de retençãos, que no sistema empregado estão separados em aproximadamente 2 minutos. Pigmentos com tempos de retenção e características espectrais semelhantes, como por exemplo luteína e zeoxantina, não foram possíveis de serem separados, como mostra a cromatografia de Dunaliella tertiolecta.

Além daqueles pigmentos descritos para as algas analisadas, outros picos, referentes a produtos de degradação foram identificados nos extratos. Estes picos provavelmente possuem duas origens: podem ser produtos da degradação dos pigmentos, ocorrida entre a filtração da cultura de algas e a análise, principalmente durante o transporte dos pigmentos, ou serem artefato da extração por acetona. A ocorrência de clorofilídeos, por exemplo, está relacionada a presença da enzima clorofilase que é ativada na presença de acetona (Mantoura \& Llewellin, 1983). A extração em acetona aquosa $90 \%$, neste

Tabela 2 - Principais pigmentos identificados nos diferentes grupos de alga, apresentados segundo a ordem de eluição, tempos de retenção (tr) e comprimento de onda dos máximos de absorção observados.

\begin{tabular}{|c|c|c|c|c|c|c|c|c|}
\hline H & PIGMENTOS & $\begin{array}{c}\mathrm{tr} \\
(\mathrm{min})\end{array}$ & $\begin{array}{l}E_{\max } \\
(\mathrm{nm})\end{array}$ & $\begin{array}{l}\text { A. carterae } \\
\text { Dinoflagelado }\end{array}$ & $\begin{array}{l}\text { D. tertiolecta } \\
\text { Alga verde }\end{array}$ & $\begin{array}{c}\text { Hillea sp } \\
\text { Criptomonada }\end{array}$ & $\begin{array}{l}\text { P. lutheri } \\
\text { Primnesiofita }\end{array}$ & $\begin{array}{l}\text { P. tricornutum } \\
\text { Diatomácea }\end{array}$ \\
\hline 1 & clorofila $\mathrm{C}_{2}$ & 5,05 & 448 & + & & + & & \\
\hline n & clorofila $\mathrm{C}_{1}+\mathrm{C}_{2}$ & 5,05 & 446 & & & & + & + \\
\hline 3 & peridinina & 7,44 & 472 & + & & & & \\
\hline 4 & fucoxantina & 8,30 & 450 & & & & + & + \\
\hline 5 & 9 cis-neoxantina & 8,76 & $437 / 464$ & & + & & & \\
\hline 0 & cis-fucoxantina & 9,42 & 439 & & & & & \\
\hline 7 & violaxantina & 9,54 & $439 / 468$ & & + & & & \\
\hline i) & dinoxantina & 10,20 & $441 / 468$ & + & & & & \\
\hline 0 & diadinoxantina & 10,40 & $446 / 474$ & + & & & + & + \\
\hline 10 & anteroxantina & 10,96 & $446 / 474$ & & + & & & \\
\hline 11 & aloxantina & 11,64 & $452 / 476$ & & & + & & \\
\hline 18 & monadoxantina & 11,99 & $446 / 472$ & & & + & & \\
\hline 10 & diatoxantina & 12,10 & & + & & & + & + \\
\hline a & luteina & 12,30 & $446 / 470$ & & + & & & \\
\hline 15 & zeoxantina & 12,64 & 452 & & + & & & \\
\hline 10 & clorofila $b$ & 11,15 & 458 & & + & & & \\
\hline 17 & crocoxentina & 15,86 & $448 / 474$ & & & + & & \\
\hline 18. & clorofila a & 16,15 & 429 & + & + & + & + & + \\
\hline 10 & $\begin{array}{c}\text { carotenoides } \\
\text { (vários) }\end{array}$ & 18,66 & & + & + & & + & + \\
\hline
\end{tabular}

Produtos de degradação (a serem confirmados*): clorofilideo $b^{*}\left(a\right.$, tr:1,60); clorofilideo $a^{*}$ (b, rt: 2,79$)$ feoforbideo $a^{*}(\mathrm{c}, \mathrm{rt}: 6,83)$; feoforbideo $a^{*}(\mathrm{~d}$, tr: 7.50$)$; alômero de cla a (e, rt: 15,69) epimero de cla a (f, tr: 16,46) e feofitina a (h, ri: 17,68). 


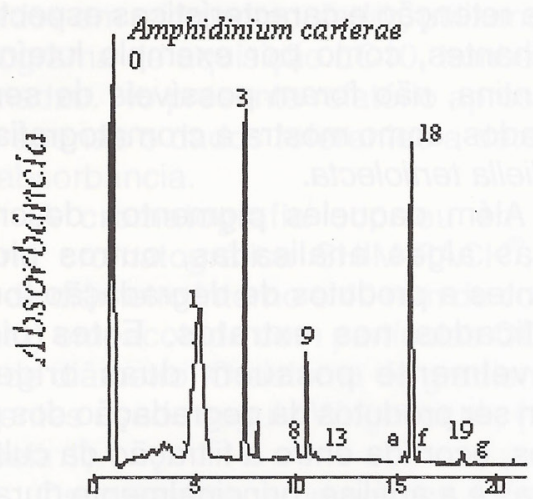

Tempo (minutos)
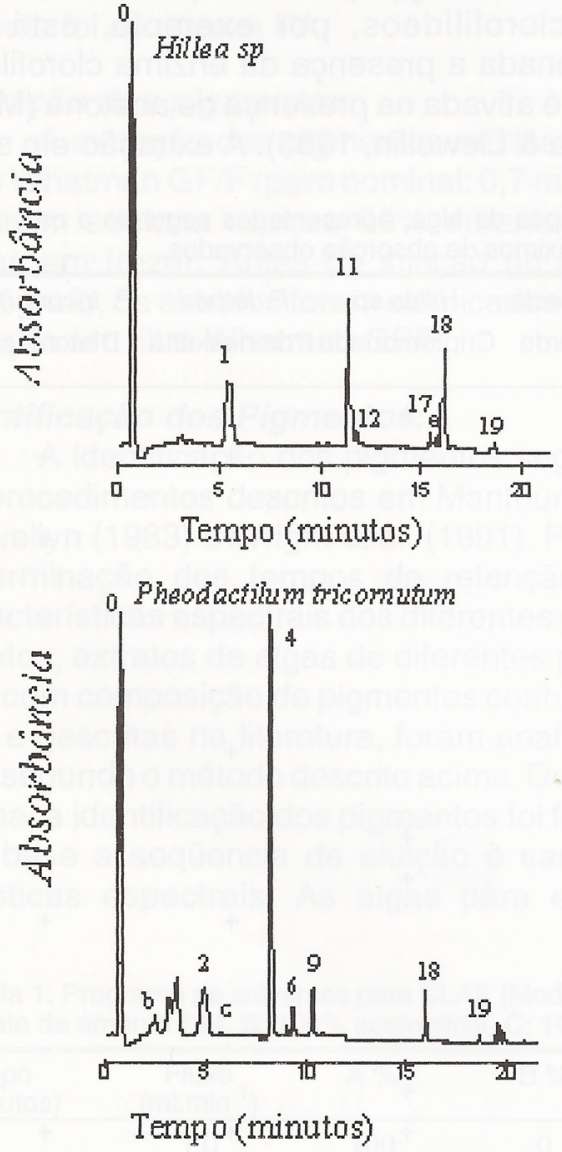

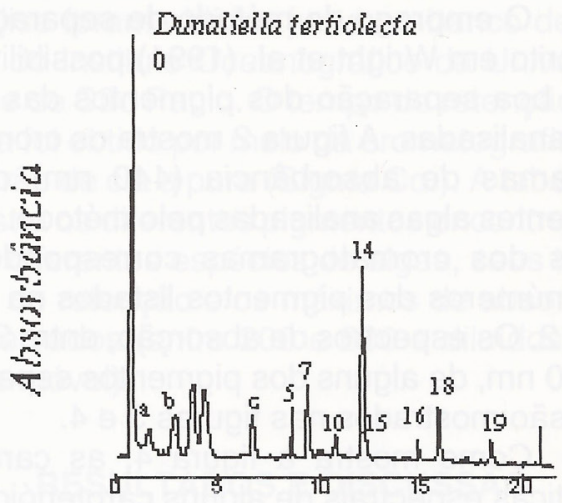

Tempo(minutos)
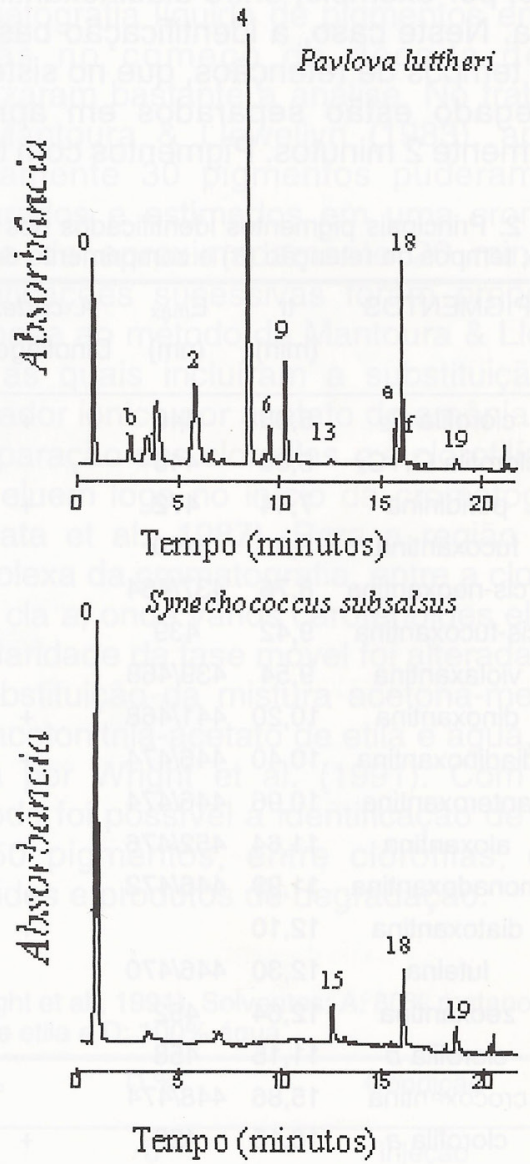

Figura 2 - Separação dos pigmentos de culturas unialgais de 6 grupos diferentes de algas. Dinoflagelado (Amphidinium carterae), alga verde (Dunaliella tertiolecta), criptomonada (Hillea sp), primnesiofita (Pavlova luthen), diatomácea (Phaeodactilum tricomutum) e cianofita (Synechococcus subsalsus). Os números dos pigmentos referem-se a tabela 2. 


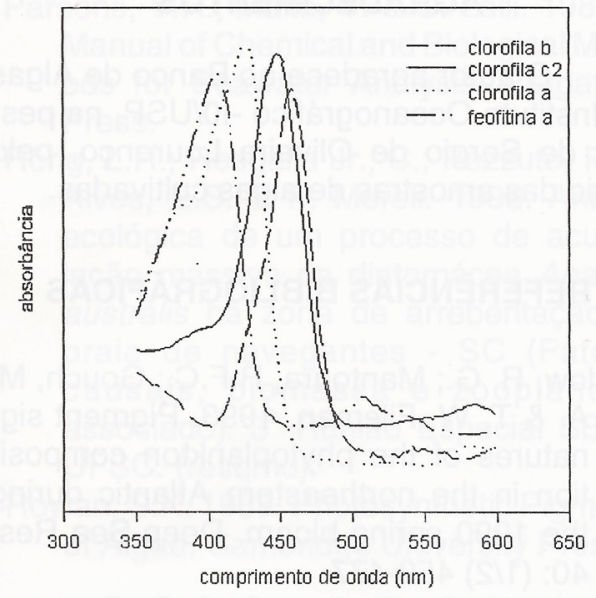

Figura 3: Espectros de absorbância, entre 350 e 600 $\mathrm{nm}$, de clorofila $c 2$ (Amphidinium carterae), clorofila $b$ (Dunaliella tertiolecta), clorofila a e feofitina a.

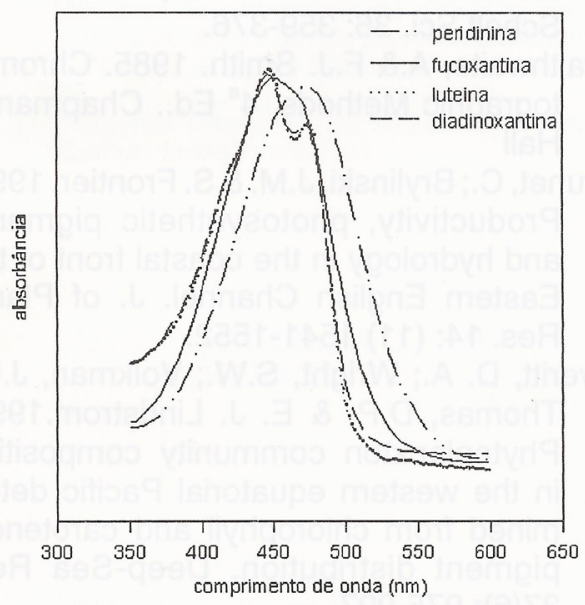

Figura 4 - Espectros de absorbância, entre 350 e 600 $\mathrm{nm}$, de alguns pigmentos identificados (peridinina de Amphidinium carterae, fucoxantina de Phaeodactilum tricomutum, luteína de Dunaliella tertiolecta e dia dinoxantina de $P$. tricomutum)

caso, reduz a atividade da clorofilase.

A figura 5 mostra a cromatografia de 3 amostras coletadas em diferentes con-dições: acumulação de Anaulus sp na praia de Navegantes (Navegantes-SC, abril de 1996), mancha de Thrichodesmium sp naenseada de Armação do Itapocoroy (Penha- SC, verão de 1996) e amostra de algas da enseada de Armação do Itapocoroy (Penha- SC, agosto de 1996). A cromatografia das florações de Anaulus sp e Thrichodesmium sp indicam florações de um mesmo grupo de algas para os dois casos, nos quais as xantofilas mais abundantes foram fucoxantina e zeoxantina, respectivamente. A cromatografia de Anaulus $\mathrm{sp}$ mostra a baixa concentração relativa do pigmento diadinoxantina. Diadinoxantina forma com diatoxantina um par de pigmentos intercambiáveis por meio da atividade de uma enzima oxidase. Baixa concentração de luz induz a epoxidação, convertendo diatoxantina a diadinoxantina. Altas concentrações de luz, por sua vez, induzem a deepoxidação (Brunet et al., 1992). A baixa concentração de diadinoxantina pode estar indicando uma fotoadaptação de Anaulus sp às condições de acumulação na zona de arrebentação da praia de Navegantes, evento registrado por Rõrig et al. (1996). Esta possibilidade necessita ser propriamente investigada, uma vez que a concentração de diatoxantina foi também baixa. A amostra de algas da enseada de Armação do Itapocoroy (Penha-SC, agosto de 1996) apresenta uma distribuição de pigmentos típica de diatomaceas. A dominância de diatomáceas na amostra foi confirmada por examinação microscópica.

Os resultados apresentados mostram a potencialidade da técnica para aplicação em amostras naturais, especialmente amostras de difícil análise microscópica, tais como aquelas de água com altos teores de material em suspensão (amostras de estuários, por exemplo), amostras de sedimentos superficiais ou ainda para a detecção da presença de algas do nano ou picoplâncton. Os trabalhos futuros se concentrarão na calibração quantitativa dos pigmentos. Esta calibração será feita, para aqueles pigmentos para os quais não existem padrões disponíveis, por meio de isolamento e determinação de sua concentração por espectroscopia. 

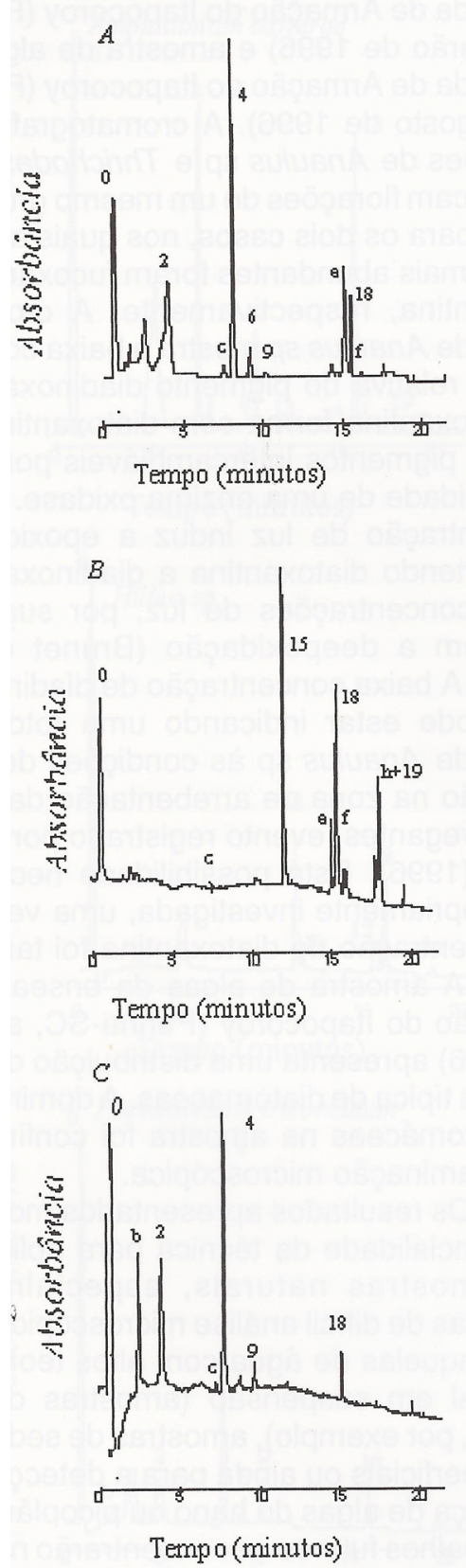

Figura 5 - Separação de pigmentos de 3 amostras naturais. Acumulação de Anaulus sp na praia de navegantes (Navegantes-SC, abril de 1996) (A), mancha de Thrichodesmium sp na enseada de Armação do Itapocoroy (Penha-SC, verão de 1996) (B), amostra de algas da enseada de Armação do Itapocoroy (PenhaSC, agosto de 1996) (C). Os números dos pigmentos referem-se a tabela 2 .

\section{AGRADECIMENTOS}

O autor agradece ao Banco de Algas do Instituto Oceanográfico -10/USP, na pessoa de Sergio de Oliveira Lourenço, pelo envio das amostras de algas cultivadas.

\section{REFERÊNCIAS BIBLIOGRÁFICAS}

Barlow, R. G.; Mantoura, R.F.C.; Gouch, M. A. \& T. W. Fileman. 1993. Pigment signatures of the phytoplankton composition in the northeastern Atlantic during the 1990 spring bloom. Deep-Sea Res. 40: (1/2) 459-477.

Bianchi, T. S.; Findlay, S. \& R. Dawson. 1993. Organic matter sources in the water column and sediments of the Hudson River Estuary: The use of plant pigments as tracers. Estuar., Coastal and Schelf Sci. 36: 359-376.

Braithwaite, A.\& F.J. Smith. 1985. Chromatographic Methods, 4a Ed.. Chapman \& Hall

Brunet, C.; Brylinski, J.M. \& S. Frontier. 1992. Productivity, photosynthetic pignnents and hydrology in the coastal front of the Eastern English Channel. J. of Plank. Res. 14: (11) 1541-1552.

Everitt, D. A.; Wright, S.W.; Volkman, J.K.; Thomas, D.P. \& E. J. Lindstrom.1990. Phytoplankton community composition in the western equatorial Pacific determined from chlorophyll and carotenoid pigment distribution. Deep-Sea Res. 37(6): 975-997.

Gieskes, W. \& G. W. Kraay. 1983. Unknown chlorophyll a derivates in the North Sea and the tropical Atlantic Ocean revealed by HPLC analysis. Limnol. Oceanogr. 28(4):757-766.

Mantoura, R. F. C. \& C. A. Llewellyn. 1983. The rapid deternnination of algal chlorophyll and carotenoid pigments and their breakdown products in natural waters by reverse-phase-high-performance 
liquid chromatography. Anal. Chim. Acta. 151:297-314.

Parsons, Y.R.; Malta, Y. \& C. Lalli. 1984. A Manual of Chemical and Biological Methods for Seawater Analysis. Pergamon Press.

Rõrig, L.R.; Resgalla Jr., C.; Pezzuto, P.R.; Alves, E.S. \& F. Moreli. 1996. Análise ecológica de um processo de acumulação massiva da diatomácea Anaulus australis na zona de arrebentação da praia de navegantes - SC (Fatores causais, biomassa e zooplâncton associado). 3' Runião Especial SBPC, UFSC. (resumo).
Rowan, S.K. 1989. Photosynthetic Pigments of Algae. Cambridge University Press.

Wright, S. W.; Jeffrey, S.W.; Mantoura, R.F.C.; Llewellyn, C.A.; Bjornland, T.; Repeta, D. \& N. Welshmeyer. 1991. Improved HPLC method for the analysis of chlorophylls and carotenoids from marine samples. Mar. Ecol. Prog. Ser.77:183-196.

Zapata, M., A.; Ayala, M.; Franco, J.M. \& J. L. Garrido. 1987. Separation of chlorophylls and their degradation products in marine phytoplankton by revesed-phase highperformance liquid chromatography. Chromatographia 23(1):26-30. 
PROENÇA: Separação de pigmentos. 